PROCEEDINGS OF THE

AMERICAN MATHEMATICAL SOCIETY

Volume 128, Number 4, Pages 1031-1037

S 0002-9939(99)05085-6

Article electronically published on July 28, 1999

\title{
GEOMETRICAL SIGNIFICANCE OF THE LÖWNER-HEINZ INEQUALITY
}

\author{
E. ANDRUCHOW, G. CORACH, AND D. STOJANOFF
}

(Communicated by David R. Larson)

Dedicated to Mischa Cotlar, with affection and admiration, on his 86th anniversary

\begin{abstract}
It is proven that the Löwner-Heinz inequality $\left\|A^{t} B^{t}\right\| \leq\|A B\|^{t}$, valid for all positive invertible operators $A, B$ on the Hilbert space $\mathcal{H}$ and $t \in[0,1]$, has equivalent forms related to the Finsler structure of the space of positive invertible elements of $\mathcal{L}(\mathcal{H})$ or, more generally, of a unital $C^{*}$ algebra. In particular, the Löwner-Heinz inequality is equivalent to some type of "nonpositive curvature" property of that space.
\end{abstract}

\section{INTRODUCTION}

The space of positive definite matrices $M_{n}(\mathbb{C})^{+}$is a well known Riemannian manifold with nonpositive curvature. It turns out that, for every unital $C^{*}$-algebra $\mathcal{A}$, the space $\mathcal{A}^{+}$of all positive invertible elements of $\mathcal{A}$ has a very rich Finsler (non-Riemannian) structure (see [14, [4], [5], [6], [15]). In this paper we show that the so-called Löwner-Heinz inequality

$$
\left\|A^{t} B^{t}\right\| \leq\|A B\|^{t} \quad\left(A, B \in \mathcal{L}(\mathcal{H})^{+}, t \in[0,1]\right)
$$

plays a central role in this geometrical study.

Recall that the inequality is a by-product of Löwner's characterization of operator monotone functions [13] and it has many applications in spectral theory [7]. Recently, Fujii, Furuta and Nakamoto [8] proved that the Löwner-Heinz inequality is equivalent to a more technical one (see (2.4)) which appeared in [6] as a corollary of a geometrical property of $\mathcal{A}^{+}$, namely the fact that the distance between two geodesics $\gamma(t), \delta(t)$ in $\mathcal{A}^{+}$is a convex function of $t$.

We show that all these assertions are, indeed, equivalent. It is known that, for Riemannian manifolds, the convexity of the function $d(\gamma(t), \delta(t))$ for any pair of geodesics $\gamma, \delta$ is equivalent to the nonpositivity of the sectional curvature. Thus, the Löwner-Heinz inequality appears as the analytic form of this geometrical behaviour of $\mathcal{A}^{+}$. In this sense, $\mathcal{A}^{+}$offers a beautiful infinite dimensional example of what Gromov calls a "espace de longueur" [10] Ch. 1], and the Löwner-Heinz inequality is the expression of the "nonpositive curvature" of $\mathcal{A}^{+}$.

Received by the editors May 29, 1997 and, in revised form, May 18, 1998.

1991 Mathematics Subject Classification. Primary 46L05, 58B20.

The authors were partially supported by UBACYT EX 261, PIP CONICET 4463/96 and PICT 2259 ANPCYT (Argentina). 
The paper contains also some results relating the geodesic distance to the work of Nussbaum [14], [15] on the part metric or Thompson distance on $\mathcal{A}^{+}$, with its corresponding notion of geodesics.

\section{Equivalent INEQUALITIES}

Theorem 1. Let $A$ be a unital $C^{*}$-algebra. Then each of the following properties holds and is equivalent to each other:

(1.1) $\left\|a^{t} b^{t}\right\| \leq\|a b\|^{t} \quad$ for all $a, b \in \mathcal{A}^{+}$and $t \in[0,1]$;

(1.2) $\left\|\log \left(a^{-t / 2} b^{t} a^{-t / 2}\right)\right\| \leq t\left\|\log \left(a^{-1 / 2} b a^{-1 / 2}\right)\right\|$ for all $a, b \in \mathcal{A}^{+}$and $t \in[0,1]$;

(1.3) For all $a_{1}, a_{2}, b_{1}, b_{2} \in \mathcal{A}^{+}$and $t \in[0,1]$,

$$
\begin{aligned}
& \left\|\left(a_{1}^{1 / 2}\left(a_{1}^{-1 / 2} b_{1} a_{1}^{-1 / 2}\right)^{t} a_{1}^{1 / 2}\right)^{1 / 2}\left(a_{2}^{1 / 2}\left(a_{2}^{-1 / 2} b_{2} a_{2}^{-1 / 2}\right)^{t} a_{2}^{1 / 2}\right)^{-1 / 2}\right\| \\
& \leq\left\|a_{1}^{1 / 2} a_{2}^{1 / 2}\right\|^{1-t}\left\|b_{1}^{1 / 2} b_{2}^{1 / 2}\right\|^{t} .
\end{aligned}
$$

Proof. The first one is the Löwner-Heinz inequality. A short proof of it can be found in [7] or [9]. The second equality has been proven in [5] as a consequence of (1.1). The third inequality has been proven in [6] and recently it has been shown in [8] that it is equivalent to (1.1). Thus, it suffices to prove that (1.2) implies (1.3). For this, take $a, b \in \mathcal{A}^{+}$. By a compactness argument, there exists $k>0$ such that

$$
a^{t}(k b)^{2 t} a^{t}>1 \quad \text { for all } t \in[0,1] \text {. }
$$

Observe that $\|\log c\|=\max \left\{\log \|c\|, \log \left\|c^{-1}\right\|\right\}$ for all $c \in \mathcal{A}^{+}$and that $\|\log c\|=$ $\log \|c\|$ for $c>1$. Then

$$
\begin{aligned}
\log \left\|a^{t}(k b)^{2 t} a^{t}\right\| & =\left\|\log \left(a^{t}(k b)^{2 t} a^{t}\right)\right\| \\
& \leq t\left\|\log \left(a(k b)^{2} a\right)\right\| \\
& =t \log \left\|a(k b)^{2} a\right\|
\end{aligned}
$$

where we use inequality (1.2) replacing $a, b$ by $a^{-2}, b^{2}$, respectively. Thus,

$$
\log \left\|a^{t} b^{2 t} a^{t}\right\|+\log k^{2 t} \leq t \log \left\|a b^{2} a\right\|+t \log k^{2}
$$

so that $\left\|a^{t} b^{t}\right\|^{2} \leq\|a b\|^{2 t}$, which is (1.1).

\section{THE GEOMETRICAL INTERPRETATION}

We give now a geometrical interpretation of the theorem. For this, we present a short description of the differential geometry of $\mathcal{A}^{+}$(see [4], [5], [6], [2] for a complete treatment). Let $\mathcal{A}$ be a unital $C^{*}$-algebra, $\mathcal{A}^{\times}$the group of invertible elements of $\mathcal{A}, \mathcal{A}_{s}$ the (real) subspace of Hermitian elements of $\mathcal{A}$. $\mathcal{A}^{+}$is an open convex subset of $\mathcal{A}_{s}$ so that it will be considered as an open submanifold of $\mathcal{A}_{s}$ and the tangent spaces $\left(T \mathcal{A}^{+}\right)_{a}$ will be identified to $\mathcal{A}_{s}$ for all $a \in \mathcal{A}^{+}$. There is a natural action of $A^{\times}$over $\mathcal{A}^{+}$given by $(g, a) \mapsto g a g^{*}\left(g \in A^{\times}, a \in \mathcal{A}^{+}\right)$. This is a transitive action: given $a, b \in \mathcal{A}^{+} g=b^{1 / 2} a^{-1 / 2}$ verifies $g a g^{*}=b$. For every $a \in A^{+}$, the map $\tau_{a}: \mathcal{A}^{\times} \rightarrow \mathcal{A}^{+} \tau_{a}(g)=g a g^{*}$ is a principal fibre bundle with a natural connection.

The covariant derivative of a tangent field $X$ along a curve $\gamma$ in $\mathcal{A}^{+}$is given by

$$
\frac{D X}{d t}=\dot{X}=\frac{1}{2}\left(X \gamma^{-1} \dot{\gamma}+\dot{\gamma} \gamma^{-1} X\right) \text {. }
$$

The curve $\gamma$ is a geodesic of the connection if $\frac{D \dot{\gamma}}{d t}=0$, i.e., if $\ddot{\gamma}=\dot{\gamma} \gamma^{-1} \dot{\gamma}$. 
In turns out that in this case $g \gamma g^{*}$ is also a geodesic for all $g \in \mathcal{A}^{\times}$. The unique geodesic $\gamma$ such that $\gamma(0)=a$ and $\dot{\gamma}(0)=X \in \mathcal{A}_{s}$ is

$$
\begin{aligned}
\gamma(t) & =e^{(t / 2) X a^{-1}} \\
& =a^{1 / 2} e^{t a^{-1 / 2} X a^{-1 / 2}} a^{1 / 2}
\end{aligned}
$$

and for every $a, b \in \mathcal{A}^{+}$there is a unique geodesic $\gamma_{a, b}$ such that $\gamma_{a, b}(0)=a$ and $\gamma_{a, b}(1)=b$, namely,

$$
\gamma_{a, b}(t)=a^{1 / 2}\left(a^{-1 / 2} b a^{-1 / 2}\right)^{t} a^{1 / 2},
$$

corresponding to $X=a^{1 / 2} \log \left(a^{-1 / 2} b a^{-1 / 2}\right) a^{1 / 2}$.

Thus, the exponential map $\exp _{a}:\left(T A^{+}\right)_{a} \rightarrow \mathcal{A}^{+}$is defined by

$$
\exp _{a}(X)=e^{(1 / 2) X a^{-1}} a e^{(1 / 2) a^{-1} X}=a^{1 / 2} e^{a^{-1 / 2} X a^{-1 / 2}} a^{1 / 2}
$$

and admits a global inverse given by

$$
\log _{a}(c)=a^{1 / 2} \log \left(a^{-1 / 2} c a^{-1 / 2}\right) a^{1 / 2} .
$$

Even if $\mathcal{A}^{+}$is not a Riemannian manifold, there is a natural Finsler structure on $\mathcal{A}^{+}$defined as follows: for $X \in\left(T \mathcal{A}^{+}\right)_{a}=\mathcal{A}_{s}$ define $\|X\|_{a}=\left\|\lambda^{-1 / 2} X \lambda^{-1 / 2}\right\|$. If $\mathcal{A}$ is represented in a Hilbert space $\mathcal{H},\|X\|_{a}$ is the norm of the symmmetric sesquilinear form $B_{X}: \mathcal{H}_{a} \times \mathcal{H}_{a} \rightarrow \mathbb{C}, B_{X}(\xi, \eta)=\langle X \xi, \eta\rangle$, where $\mathcal{H}_{a}$ is $\mathcal{H}$ with the scalar product $\langle\xi, \eta\rangle_{a}=\langle a \xi, \eta\rangle$. If $g \in \mathcal{A}^{\times}$, then $g: \mathcal{H}_{\text {gag }} \rightarrow \mathcal{H}_{a}$ is an isometry, so that

$$
\left\|B_{g X g^{*}}\right\|=\left\|B_{X}\right\| \quad \text { and }\left\|g X g^{*}\right\|_{g a g^{*}}=\|X\|_{a} .
$$

It can be shown that the geodesic $\gamma_{a, b}$ is shortest among all curves $\gamma:[0,1] \rightarrow \mathcal{A}^{+}$ such that $\gamma(0)=a$ and $\gamma(1)=b$, where the length of $\gamma$ is

$$
\ell(\gamma)=\int_{0}^{1}\|\dot{\gamma}(t)\|_{\gamma(t)} d t
$$

Thus, if we define the geodesic or rectifiable distance $d$ by

$$
d(a, b)=\inf \left\{\text { length } \gamma: \gamma \text { is a smooth curve in } A^{+} \text {joining } a \text { and } b\right\},
$$

then $d(a, b)=$ length $\gamma_{a, b}$. Since $\left\|g X g^{*}\right\|_{g a g^{*}}=\|X\|_{a}$ for all $g \in \mathcal{A}^{\times}, a \in \mathcal{A}^{+}$and $X \in\left(T A^{+}\right)_{a}$, it follows that $d\left(g a g^{*}, g b g^{*}\right)=$ length $\gamma_{g a g^{*}, g b g^{*}}=$ length $\gamma_{a, b}=$ $d(a, b)$; thus, $\mathcal{A}^{\times}$acts transitively and isometrically on $\mathcal{A}^{+}$, and for computations one can usually transport the situation to $a=1$. Observe also that the geodesics with origin 1 have the form $\gamma_{1, a}(t)=a^{t}$.

It is easy to verify that $d(a, b)=\left\|\log a^{-1 / 2} b a^{-1 / 2}\right\|$ so that, when $a$ commutes with $b, d(a, b)=\left\|\log b a^{-1}\right\|$.

Remark 1. We shall prove later that the geodesic distance on $\mathcal{A}^{+}$coincides with the so-called Thompson or part metric so that the results of Nussbaum [4] are still valid.

Remark 2. A relevant property of the geodesic distance on $\mathcal{A}^{+}$is that the metric space $\left(\mathcal{A}^{+}, d\right)$ is complete. A proof of this statement can be found in [14], where Nussbaum uses the Thompson metric. This fact, among many others, justifies the introduction of this distance. On the other hand, as observed by Vesentini [18], the 
relevance of $d$ is related to the fact that the Haar measure of the locally compact group $\mathbb{C}^{+}=\{t \in \mathbb{R}: t>0\}$ is determined, up to a constant, by

$$
m(\alpha, \beta)=|\log \beta-\log \alpha|=\left|\log \frac{\beta}{\alpha}\right|
$$

for any interval $(\alpha, \beta)$.

The next result shows that the Löwner-Heinz inequality is equivalent to two geometrical properties which characterize Riemannian manifolds with nonpositive curvature. We shall make some comments on this after the proof of the theorem.

Theorem 2. If $\mathcal{A}$ is a unital $C^{*}$-algebra, then the following properties hold and are equivalent:

(2.1) $\left\|a^{t} b^{t}\right\| \leq\|a b\|^{t}$ for every $a, b \in \mathcal{A}^{+}$and $t \in[0,1]$;

$(2.2) d\left(a^{t}, b^{t}\right) \leq t d(a, b)$ for every $a, b \in \mathcal{A}^{+}$and $t \in[0,1]$;

(2.3) for every pair of geodesics of $\mathcal{A}^{+}$the real function

$$
t \mapsto d(\gamma(t), \delta(t))
$$

is convex.

Proof. Observe that (2.2) is the rewriting of inequality (1.2), using the explicit expression for $d$. On the other hand, (1.3) is an easy consequence of (2.3) (see [6], where there is also a proof of (2.3) by a completely different method). Thus, it suffices to show that (1.3) implies property (2.3). Observe that (2.3) is equivalent to the inequality

$$
\left\|\log \left(\delta(t)^{-1 / 2} \gamma(t) \delta(t)^{-1 / 2}\right)\right\| \leq(1-t)\left\|\log \left(a_{1}^{-1 / 2} a_{2} a_{1}^{-1 / 2}\right)\right\|+t\left\|\log \left(b_{1}^{-1 / 2} b_{2} b_{1}^{-1 / 2}\right)\right\|
$$

for every $t \in[0,1]$, where $\gamma$ joins $a_{1}$ and $b_{1}$ and $\delta$ joins $a_{2}$ and $b_{2}$. The proof that (2.4) follows from (1.3) uses the same trick of Theorem 1. After changing $\gamma$ by $k \gamma$ for a convenient $k>0$ we may suppose that

$$
\delta(t)^{-1} \gamma(t) \delta(t)^{-1 / 2} \geq 1
$$

for every $t \in[0,1]$, so that

$$
\begin{aligned}
\left\|\log \left(\delta(t)^{-1 / 2} \gamma(t) \delta(t)^{-1 / 2}\right)\right\| & =\log \left\|\delta(t)^{-1 / 2} \gamma(t) \delta(t)^{-1 / 2}\right\| \\
& \leq \log \left(\left\|a_{1}^{-1 / 2} a_{2} a_{1}^{-1 / 2}\right\|^{1-t}\left\|b_{1}^{-1 / 2} b_{2} b_{1}^{-1 / 2}\right\|^{t}\right) \\
& =(1-t) \log \left\|a_{1}^{-1 / 2} a_{2} a_{1}^{-1 / 2}\right\|+t \log \left\|b_{1}^{-1 / 2} b_{2} b_{1}^{-1 / 2}\right\| \\
& =(1-t)\left\|\log \left(a_{1}^{-1 / 2} a_{2} a_{1}^{-1 / 2}\right)\right\|+t\left\|\log \left(b_{1}^{-1 / 2} b_{2} b_{1}^{-1 / 2}\right)\right\|
\end{aligned}
$$

which ends the proof.

Remarks. In Gromov's lectures [1] one finds two characterizations of Riemannian manifold with nonpositive curvature. The first one rests on the local diffeomorphism property of the exponential map: if $M$ is a Riemannian manifold and $r \in M$, the exponential map $\exp _{r}:(T M)_{r} \rightarrow M$ is a local diffeomorphism so that in the neighborhood of $r$ there is a map defined by

$$
\rho_{t}(m)=\exp _{r}\left(t \exp _{r}^{-1}(m)\right) \quad(t \in[0,1]) ;
$$


then $M$ has nonpositive curvature if and only if for every $t \in[0,1]$ and $m_{1}, m_{2}$ close to $r$

$$
d\left(\rho_{t}\left(m_{1}\right), \rho_{t}\left(m_{2}\right)\right) \leq t d\left(m_{1}, m_{2}\right) .
$$

This expression reduces, in the case when $M$ is the (non-Riemannian!) manifold $\mathcal{A}^{+}$, to the expression

$$
d\left(r^{1 / 2}\left(r^{-1 / 2} m_{1} r^{-1 / 2}\right)^{t} r^{1 / 2}, r^{1 / 2}\left(r^{-1 / 2} m_{2} r^{-1 / 2}\right)^{t} r^{1 / 2}\right) \leq t d\left(m_{1}, m_{2}\right)
$$

which is exactly (2.2), with $m_{1}=a, m_{2}=b$, due to the fact that $\mathcal{A}^{\times}$acts isometrically over $\mathcal{A}^{+}$. Returning to Gromov's lectures [1], one can find there a characterization of Riemannian manifolds with nonpositive curvature as those Riemannian manifolds so that the distance $d(\gamma(t), \delta(t))$, for two geodesics $\gamma, \delta$, is a convex function of $t$. Thus, Theorem 2 shows that the Löwner-Heinz inequality means exactly that $\mathcal{A}^{+}$has this type of "negatively curved space" behaviour. Recall that this is not the first time that a classical inequality appears as describing a geometrical behaviour of $\mathcal{A}^{+}$: in [3] it has been shown that Segal's inequality

$$
\left\|e^{X+Y}\right\| \leq\left\|e^{X / 2} e^{Y} e^{X / 2}\right\|
$$

valid for Hermitian operators $X, Y\left[16\right.$ is equivalent to the property that $\exp _{a}$ increases distances:

$$
d\left(\exp _{a} X, \exp _{a} Y\right) \geq\|X-Y\|_{a}
$$

for all $X, Y \in\left(T \mathcal{A}^{+}\right)_{a}$; in Riemannian geometry this happens, again, in nonpositive curvature manifolds. The reader may find a deduction of Segal's inequality from Löwner-Heinz's in [2]; see also [6].

The part metric (or Thompson metric [17]) of $\mathcal{A}^{+}$can be defined as

$$
d_{p}(a, b)=\max \{\log \inf \{\alpha>0: a \leq \alpha b\}, \log \inf \{\beta>0: b \leq \beta a\}\} .
$$

Nussbaum 14 proves that

$$
d_{p}(a, b)=\max \left\{\log \left\|a^{-1 / 2} b a^{-1 / 2}\right\|, \log \left\|b^{-1 / 2} a b^{-1 / 2}\right\|\right\} .
$$

Let us show that $d_{p}$ coincides with the distance $d$ obtained by differential geometric methods.

Proposition. For every $a, b \in A^{+}$

$$
d_{p}(a, b)=\left\|\log a^{-1 / 2} b a^{-1 / 2}\right\| .
$$

Proof. Recall that $\|\log c\|=\max \left\{\log \|c\|, \log \left\|c^{-1}\right\|\right\}$ for all $c \in \mathcal{A}^{+}$. Then

$$
\begin{aligned}
d(a, b) & =\left\|\log a^{-1 / 2} b a^{-1 / 2}\right\| \\
& =\max \left\{\log \left\|a^{-1 / 2} b a^{-1 / 2}\right\|, \log \left\|a^{1 / 2} b^{-1} a^{1 / 2}\right\|\right\}
\end{aligned}
$$

and it suffices to show that $\left\|a^{1 / 2} b^{-1} a^{1 / 2}\right\|=\left\|b^{-1 / 2} a b^{-1 / 2}\right\|$. But this is obvious, taking $x=a^{1 / 2} b^{-1 / 2}$ and using that $\left\|x^{*} x\right\|=\left\|x x^{*}\right\|=\|x\|^{2}$.

Remarks. 1) Nussbaum [14] proved that $\gamma_{a b}(t)=a^{1 / 2}\left(a^{-1 / 2} b a^{-1 / 2}\right)^{t} a^{1 / 2}$ curve which is shortest, for the part metric, among all curves joining $a$ and $b$. On the other side, Corach, Porta and Recht [4, 5, 3] proved that $\gamma_{a, b}$ is a geodesic (in the sense of the differential geometry) and is a shortest curve joining $a$ and $b$. The result above justifies the coincidence between both approaches. Thus, the convexity results obtained in the theorem above are valid also in Nussbaum's context. It 
should be noticed that Nussbaum [15] gets by a general method a whole family of shortest curves joining two fixed $a, b \in \mathcal{A}^{+}$. The geodesic $\gamma_{a, b}$ appears, thus, as a distinguished member of the family.

2) Liverani and Wojtkowski [12] got, by a different method, a similar expression for the distance $d$. There is also a previous paper by Vesentini [18] where the distance on $\mathcal{A}^{+}$can be obtained as a Carathéodory type metric.

3) Given a metric space $X$ and a map $\varphi: X \rightarrow X$, consider the dilation index of $\varphi:$

$$
\operatorname{dil}(\varphi)=\sup _{x_{1} \neq x_{2}} \frac{d\left(\varphi\left(x_{1}\right), \varphi\left(x_{2}\right)\right)}{d\left(x_{1}, x_{2}\right)}
$$

which in analysis is usually called the best Lipschitz constant of $\varphi$. Gromov 10 uses, as a useful tool for the study of his "espaces de longueur", the notion short and strictly short maps. The map $\varphi$ is short if dil $(\varphi) \leq 1$ and is strictly short if dil $(\varphi)<1$ (classically called contractions).

Inequality (2.2) shows that the map $\varphi_{t}: a \mapsto a^{t}$ (for $t \in[0,1]$ ) verifies dil $\left(\varphi_{t}\right) \leq$ $t$. Indeed, it can easily be shown that $\operatorname{dil}\left(\varphi_{t}\right)=t$. Thus, $\varphi_{t}$ is strictly short for $0 \leq t<1$. More generally, using the isometric action of $\mathcal{A}^{\times}$over $\mathcal{A}^{+}$, it follows that, for fixed $a$, the map $b \mapsto \gamma_{a, b}(t)$ has dilation index $t$.

\section{REFERENCES}

[1] W. Ballmann, M. Gromov, V. Schroeder, "Manifolds of non positive curvature", Birkhauser, Boston-Basel-Stutgart, 1985. MR 87h:53050

[2] G. Corach, "Operator inequalities, geodesics and interpolation", Functional Analysis and Operator Theory, Banach Center Publications, Vol. 30, Polish Academy of Sciences, Warszawa, 1994, pp. 101-115. MR 95d:58009

[3] G. Corach, H. Porta, L. Recht, "A geometric interpretation of Segal's inequality $\left|e^{X+Y}\right| \leq$ $\left|e^{X / 2} e^{Y} e^{X / 2}\right| "$, Proc. Amer. Math. Soc. 115 (1992), 229-231. MR 92h:46105

[4] G. Corach, H. Porta, L. Recht, "The geometry of the space of selfadjoint invertible elements in a $C^{*}$-algebras", Integral Equations Operator Theory 16 (1993), 333-359. MR 94d:58010

[5] G. Corach, H. Porta, L. Recht, "Geodesics and operator means in the space of positive operators", Internat. J. Math. 4 (1993), 193-202. MR 94c:46114

[6] G. Corach, H. Porta, L. Recht, "Convexity of the geodesic distance on spaces of positive operators", Illinois J. Math. 38 (1994), 87-94. MR 94i:58010

[7] H.O. Cordes, "Spectral theory of linear differential operators and comparison algebras", London Mathematical Society Lecture Notes Series 76, Cambridge University Press, 1987. MR 88g:35144

[8] M. Fujii, T. Furuta, R. Nakamoto, "Norm inequalities in the Corach-Porta-Recht theory and operator means", Illinois J. Math. 40 (1996), 527-534. MR 97i:47026

[9] T. Furuta, "Norm inequalities equivalent to Löwner-Heinz theorem", Rev. Math. Phys. 1 (1989), 135-137. MR 91b:47028

[10] M. Gromov, "Structures métriques pour les variétés riemanniennes", CEDIC/Fernand Nathan, Paris, 1981. MR 85e:53051

[11] E. Heinz, "Beiträge zur Storungstheorie der Spektralzerlegung", Math. Ann. 123 (1951), 415-438. MR 13:471f

[12] C. Liverani, M.P. Wojtkowski, "Generalization of the Hilbert metric to the space of positive definite matrices", Pacific J. Math. 166 (1994), 339-355. MR 95m:53099

[13] K. Löwner, "Über monotone Matrix funktionen", Math. Z. 38 (1934), 177-216.

[14] R. Nussbaum, "Hilbert's projective metric and iterated nonlinear maps", Mem. Amer. Math. Soc. 391 (1988). MR 89m:47046]

[15] R. Nussbaum, "Finsler structures for the part metric and Hilbert's projective metric and applications to ordinary differential equations", Diff. Integral Equations 7 (1994), 1649-1707. MR 95b:58010 
[16] I. Segal, "Notes toward the construction of non linear relativistic quantum fields III", Bull. Amer. Math. Soc. 75 (1969), 1390-1395. MR 40:5217

[17] A.C. Thompson, "On certain contraction mappings in a partially ordered vector space", Proc. Amer. Math. Soc. 14 (1963), 438-443. [MR 26:6727]

[18] E. Vesentini, "Invariant metrics on convex cones", Ann. Sc. Norm. Sup. Pisa (Ser. 4) 3 (1976), 671-696. MR 55:6206

Instituto de Ciencias, Universidad Nacional de General Sarmiento, Roca 850, 1663 San Miguel, Argentina

E-mail address: eandruch@mate.dm.uba.ar

Departamento de Matemática, Facultad de Ciencias Exactas, Ciudad Universitaria, 1428-Buenos Aires, Argentina

E-mail address: gcorach@mate.dm.uba.ar

Instituto Argentino de Matemática, SaAvedra 15, 1083-Buenos Aires, Argentina

E-mail address: demetrio@mate.dm.uba.ar 